\title{
Geração de Malha com Elementos de Interface para Modelagem em Microestrutura Policristalina em 2D
}

\author{
Lino CE $\S$ \\ $\S$ Instituto Federal de Educação, Ciência e Tecnologia São Paulo, Brasil.
}

\begin{abstract}
Resumo: Para calcular elementos de interface em materiais policristalinos para modelagens bidimensionais, foi implementado um algoritmo, que realiza uma análise através de um arquivo de malha bidimensional, que modifica usando um gerador de elementos e escolhe os grãos existentes por um fator predefinido para criar elementos de interface, facilitando a modelagem de fenômenos de danos que ocorrem nos limites dos grãos. Se a malha da microestrutura policristalina em 2D original, modificando a periodicidade da malha no limite do domínio da modelagem. Essa metodologia criar automaticamente distintas estruturas granulares que permite as propriedades mecânicas de metais e outros materiais sejam modelados e simulados. A importância dessa metodologia permite que a simulação faça uma variabilidade da amostragem de fratura e das propriedades mecânicas que são observadas nos experimentos a serem reproduzidas nas simulações. A malha resultante é exportada em um formato legível por software de Métodos dos Elementos Finitos comerciais e/ou OpenSource.
\end{abstract}

\section{Palavras-chave: Malha Policristalina 2D, Microestrutura, Zona Coesiva, Interface}

\begin{abstract}
For interface calculation elements in polycrystalline materials for two-dimensional modeling, an algorithm was implemented, which performs an analysis through a twodimensional mesh file, which modifies using an element generator and chooses the existing grains by a predefined factor to create interface elements, facilitating the modeling of damage phenomena that occur at grain boundaries. If the mesh of the original 2D polycrystalline microstructure, modifying the mesh periodicity at the boundary of the modeling domain. This methodology automatically creates distinct granular structures that allow the mechanical properties of metals and other materials to be modeled and simulated. The importance of this methodology allows the simulation to make a variability of the fracture sampling and the mechanical properties that are observed in the experiments to be reproduced in the simulations. The resulting mesh is exported in a software-readable format from commercial Finite Element Methods and/or OpenSource.
\end{abstract}

Keywords: 2D Polycrystalline Mesh, Microstructure, Cohesive Zone, Interface

Introdução: A previsão precisa de falhas em sólidos é uma questão de grande relevância em diferentes setores industriais desde aeroespacial e aeronáutica até mesmo na geração de energia renovável. Na escala macroscópica, a fratura é geralmente originada pelo desenvolvimento de vários defeitos microscópicos, como os vazios, discordância, concentradores de tensão, maclas e fissuras entre outros, que crescem e coalescem levando à deterioração das propriedades aparentes do material e da capacidade de carga do componente estrutural. Em particular, na escala microscópica, a interação entre a fratura intergranular (trincas que se propagam entre os grãos) e transgranular (que se propagam através dos grãos) em materiais policristalinos é de grande preocupação na Ciência dos Materiais e aplicações de Engenharia. 
Para simular as propriedades mecânicas dos metais, requer uma análise da resposta elástica e plástica da microestrutura (1). Os metais consistem em grãos com estrutura cristalina, cujas dimensões podem ter uma forma e orientação específicas. A simulação de ensaios mecânicos pode ser realizada utilizando solvers de Elementos Finitos (3), através de softwares comerciais ou OpenSource. As subrotinas executadas pelo usuário podem ser usadas para modelar as propriedades mecânicas de diferentes grãos (4). Interfaces de elemento coesivo implementado por meio de uma subrotina pelo usuário para chapas metálicas de espessura fina, são usadas para modelar a nucleação e propagação de trincas (5-7).

O software disponível para geração de malhas policristalinas é o Neper, que é OpenSource (8). Ele pode gerar malhas policristalinas com tamanhos de grãos arbitrários, com diversas distribuições e várias orientações, ele também pode importar imagens de pesquisas experimentais. Os recursos de geração de malha policristalinas são geralmente incluídos no software mencionado acima. No entanto, as formas geométricas que podem ser geradas e as opções de malha são limitadas dependendo da geometria a ser simulada. Especificamente, uma interface gráfica do usuário que permite uma ampla variedade de opções de manipulação geométricas.

A geometria pode ser modificada usando extrusões de formas arbitrárias e várias peças podem ser montadas em um modelo, portanto, as amostras com formas arbitrárias podem ser reproduzidas. Além disso, vazios internos podem ser introduzidos. Se dispositivos externos aplicarem carga à amostra, eles podem ser incluídos no modelo. Podem ser introduzidas condições de contorno arbitrárias e contato entre as partes. Os recursos de geração de malha também são mais avançados. Diferentes tipos de elementos podem ser usados nos algoritmos para geração de malha. Portanto, vincular softwares de geração de malhas policristalinas, como o Neper representa uma grande necessidade para a comunidade Científica e de Engenharia.

A geração automatizada de malhas policristalinas também é útil ao executar um conjunto de simulações com diferentes tamanhos e formatos de grãos. Sendo assim, esse tipo de malha tende a ser de melhor forma para demostrar o fenômeno de Fadiga Mecânica e Mecânica da Fratura e as propriedades mecânicas podem ser entendidos e os limites de segurança podem ser estabelecidos durante o projeto do componente.

Limites de grãos, que são as interfaces $2 \mathrm{D}$ entre os grãos vizinhos, fornecem nucleação de trincas e locais de propagação em uma variedade de metais (9). Este fenômeno pode ser modelado usando elementos de interface, cujas propriedades mecânicas são descritas por uma lei de separação de tração (10). Os elementos da interface constituem uma malha 2D nos limites dos grãos. No entanto, alguns dos softwares comerciais de Elementos Finitos não oferecem a possibilidade de gerar automaticamente elementos coesivos para espessura fina.

O script PyPolyCris2D para geração de malha policristalina pode criar apenas elementos de interface quadrangulares e triangulares no vértice dos grãos e dentro dos grãos são usados apenas elementos triangulares. O script foi desenvolvido é especificamente para metais, mas sua aplicabilidade pode ser facilmente estendida e adaptada para os diferentes materiais, por exemplo, materiais compostos.

Motivação: $\mathrm{Na}$ literatura mostra alguns modelos simplificados específicos para fratura intergranular foram propostos em $(11,12,13,14,15)$ com base no Modelo de Zona Coesiva (CZM) inserido ao longo dos limites internos dos grãos. As formulações de fissuras coesivas podem ser entendidas como modelos fenomenológicos nos quais os eventos da fratura são desencadeados pela avaliação de uma lei particular de tração de deslocamento. Esta técnica numérica incorpora inerentemente uma escala de comprimento característica e tem sido 
amplamente utilizada para simular danos em materiais (16), embora os caminhos de trinca sejam restritos ao longo das bordas do elemento, veja uma ampla discussão em $(17,18,19)$.

No entanto, a fratura transgranular também é um modo de falha relevante em alguns materiais e, em muitos casos, é desenvolvida em competição com a falha intergranular. Portanto, o desenvolvimento de técnicas numéricas que permitem uma modelagem confiável de fissuras dos grãos, com caminhos de falha arbitrários é de fato necessários para fornecer uma ferramenta de simulação abrangente, ambos os tipos de propagação de trincas foram relatados em $(20,21)$. Nessas publicações, os autores documentaram de forma abrangente a importância de se considerar a anisotropia dos grãos no plano, que afeta notavelmente o comportamento da fratura. Uma primeira tentativa de modelar os dois tipos de fratura foi realizada (9), por uma abordagem de modelo de zona coesa intrínseca. Para evitar a dependência da malha e conformidade material não física, a abordagem extrínseca pode ser proposta, como em (10). No entanto, para materiais muito frágeis, as abordagens do modelo de zona coesa sofrem da complexidade de resolver a zona do processo, que é muito pequena.

Em contraste com os métodos numéricos anteriores, baseados apenas em modelos de zona coesiva, vários estudos também foram propostos, para explicar a propagação de trinca transgranular em policristalino por meio do Método dos Elementos Finito Estendido (XFEM) (22), em que o caminho da trinca não depende explicitamente da discretização espacial subjacente do domínio físico em elementos finitos.

Descrição do Algoritmo, Geração da Malha e Elementos. Nesta seção, serão relatadas as principais funcionalidades do script, usado para a geração de malha policristalina. Utilizando como analisador, o software Neper, mas que não será descrito em detalhes nesse artigo. Uma malha policristalina 2D é gerado usando Neper (9), como mostrado no lado esquerdo da Figura 1. É gerado com as coordenadas ( $\mathrm{x}, \mathrm{y})$, cuja os grãos estão demostrados de forma colorida. Estes são introduzidos na superfície da geometria a ser estudada. As arestas conectam os vértices dos grãos, que estão inseridos na geometria, como partições da superfície. A estrutura de grão 2D é gerada, portanto, a geração na superfície, conforme mostrado a Figura 1. A geometria é particionada em grãos por varredura as bordas, gerado na superfície do plano, ao longo de uma direção de varredura que corresponde, nulo na direção z. As propriedades mecânicas indicam a orientações dos grãos e o tipo de material. Destas formas materiais bifásicos, com grãos constituídos por diferentes estruturas cristalinas, podem ser incluídos. A seção sólida correspondente é criada e atribuída a cada grão. Finalmente, o modelo com a malha está pronto e pode ser transformado e usando e pode ser realizado o processo da geração das interfaces, como mostrado no lado direito da Figura 1. 

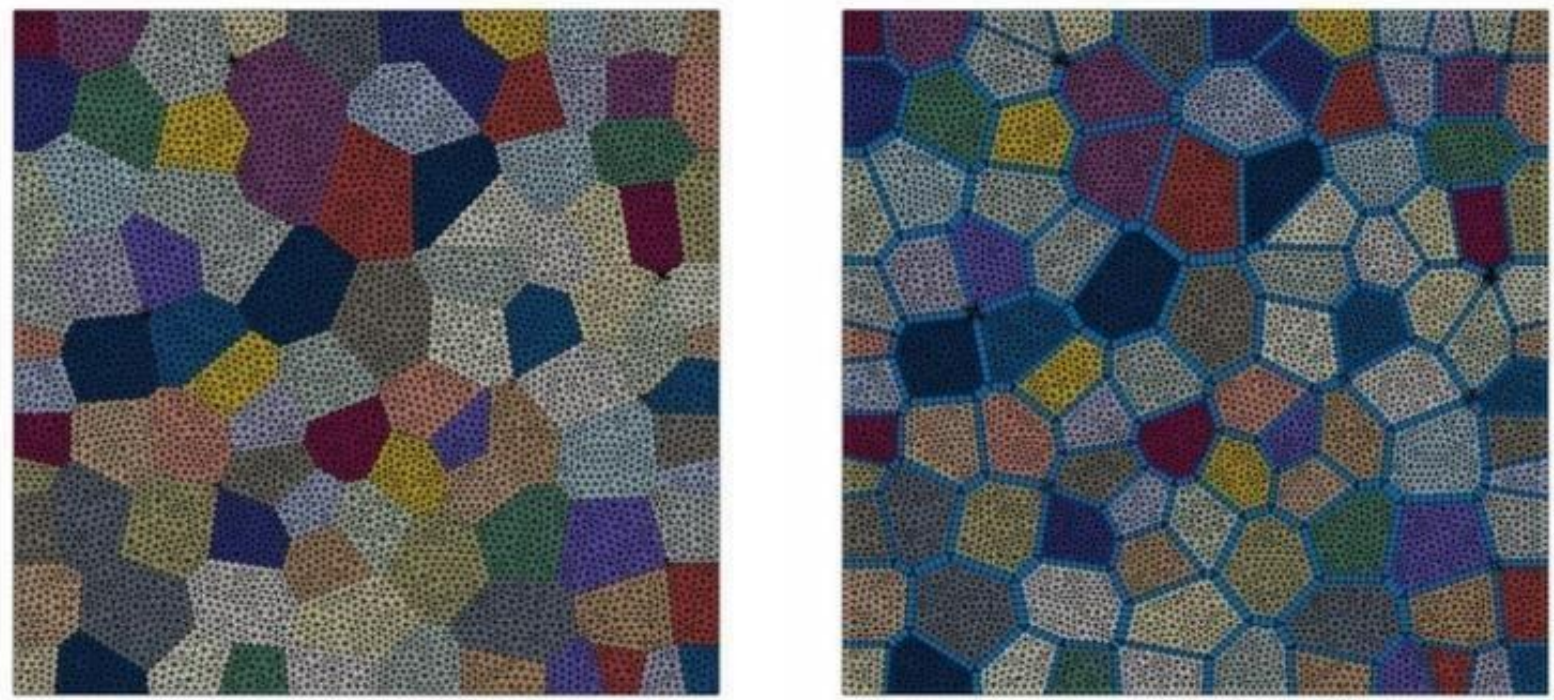

Figura 1. A imagem da esquerda está sem as interfaces, a da direita com as interfaces.

O conjunto elementos triangulares em diferentes cores representam os grãos, como mostra na Figura 2. São representados pelos elementos retangulares azuis o contorno dos grãos e em cada vértice dos grãos são representados por elementos triangulares azuis, onde cada desses elementos em massa contém 4 nós para os retangulares e 3 nós para os triangulares que correspondendo aos vértices dos grãos, que esses elementos azuis denominamos como interfaces.

Os elementos da face dos grãos são conectados por interfaces, que contém 4 nós ou 3 nós nos vértices dos grãos, e cada nó e cada elemento são caracterizados por um índice único de número inteiro positivo. Primeiro, é construída uma estrutura de dados chamada conectividade que armazena os índices de todos os elementos na face dos grãos. Os conjuntos de elementos a granel são construídos pelo software comercial e/ou opensource, que contêm todos os elementos pertencentes a um determinado grão.

Os elementos da interface são construídos na face dos elementos dos grãos. Portanto, os nós que estão nessas interfaces devem são identificados.

Para identificar nós de interface, é usada a conectividade entre os elementos da face dos grãos, um certo nó, de um dos elementos da face dos grãos. Elementos da face dos grãos adjacentes no limite de grão são identificados como elementos que contêm nós para gerar uma interface. Os pares de elementos da face dos grãos adjacentes são identificados verificando se eles compartilham de mesmo nós de interface. Como pode ser verificando no lado direito da Figura 2 , cada interface que é representado por elementos azuis, são conectados com nós de elementos de diferente grão, separados pelas interfaces.

Neste ponto, a interface contém informações sobre os 4 nós que formarão o elemento de informações sobre os 2 elementos da face dos grãos adjacentes.

Em uma simulação de elemento finito, um vetor de deformação é associado a cada nó e uma lei de tração separação é usada, que é baseada na deformação. Portanto, a ordem dos nós no elemento de interface é importante. Na deformação, outros nós virtuais coincidem com as distâncias entre esses pares de nós que determinar a separação da interface, para uma simulação de uma trinca.

Um sistema de coordenadas de referência XY é construído. No X e Y, uma perpendicular ao outro e pertence ao plano que contém os nós. 

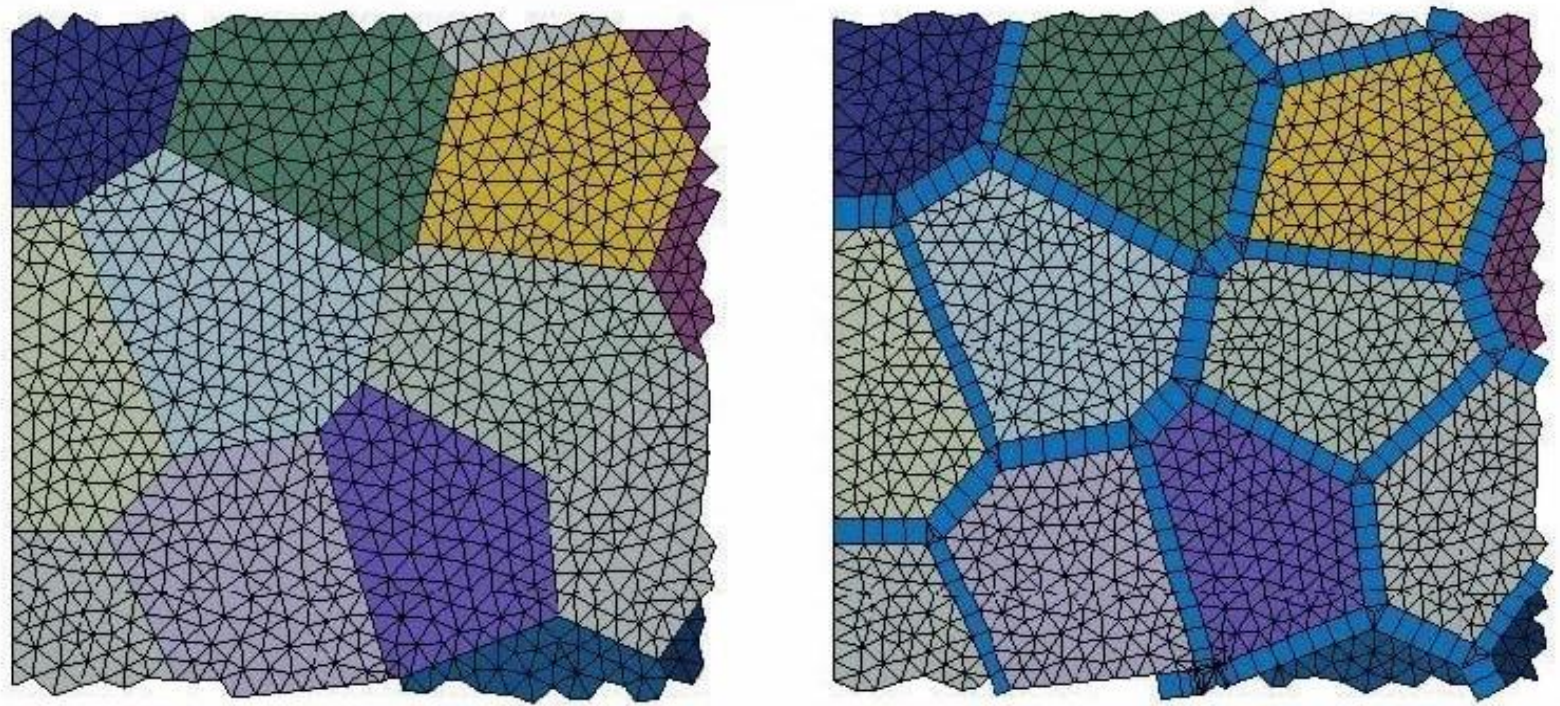

Figura 2. A imagem da esquerda está sem as interfaces, a da direita com as interfaces.

Conclusões. O script foi desenvolvido para gerar volumes representativos policristalino e elementos de interface nos contornos dos grãos. Esses recursos não estão disponíveis em software comercial e/ou opensource, mas normalmente são necessários para modelar propriedades mecânicas na escala de comprimento.

O script para geração de elementos de interface é de interesse geral. Ele representa um exemplo de detecção de interface em uma malha. A estratégia usada para encontrar a orientação dos elementos da interface, é baseado na ordenação dos nós de elemento de interface e na correspondência específica entre nós de elemento de interface e nós de elemento em massa. Ele pode ser usado para encontrar as direções que apontam para o interior e o exterior de um conjunto de elementos genéricos, com isso auxiliando nas simulações de Mecânica da Fratura e Fadiga Mecânica.

\section{Referências}

(1) Roters F, Eisenlohr P, Bieler TR, Raabe D. Crystal plasticity finite element methods in materials science and engineering. Wiley; 2010. Disponível em: <https: //onlinelibrary.wiley.com/doi/book/10.1002/9783527631483). (Acesso em 21 de Abril de 2021).

(2) Roters F, Diehl M, Shanthraj P, Eisenlohr P, Reuber C, Wong S, et al. DAMASK - the Düsseldorf advanced material simulation kit for modeling multi-physics crystal plasticity, thermal, and damage phenomena from the single crystal up to the component scale. Computational Materials Science 2018; 158:420-478.

(3) Dunne F, Rugg D, Walker A. Lengthscale-dependent, elastically anisotropic, physicallybased hcp crystal plasticity: Application to cold-dwell fatigue in Ti alloys. Int J Plast 2007; 23(6):1061-83. 
(4) Camacho G, Ortiz M. Computational modelling of impact damage in brittle materials. Int J Solids Struct 1996; 33(20):2899-938.

(5) Ortiz M, Pandolfi A. Finite deformation irreversible cohesive elements for three dimensional crack propagation analysis. Internat J Numer Methods Engrg 1999; 44(9):126782 ,

(5) Elmukashfi E, Tarleton E, Cocks ACF. A modelling framework for coupled hydrogen diffusion and mechanical behaviour of engineering components. Comput Mech 2020; 66(1):189-220, 2020

(6) Quey R, Dawson P, Barbe F. Large-scale 3D random polycrystals for the finite element method: Generation, meshing and remeshing. Comput Methods Appl Mech Engrg , 2011; 200(17):1729-45.

(7) Grilli N, Cocks AC, Tarleton E. Crystal plasticity finite element modelling of coarse-grained $\alpha$-uranium. Comput Mater Sci 2020; 171:109276.

(8) Irastorza-Landa A, Grilli N, Van Swygenhoven H. Laue micro-diffraction and crystal plasticity finite element simulations to reveal a vein structure in fatigued $\mathrm{Cu}$. J Mech Phys Solids 2017; 104:157-71.

(9) A Infuso, M Corrado, and M Paggi. Image analysis of polycrystalline solar cells and modelling of intergranular and transgranular cracking. Journal of the European Ceramic Society 2014; 34(11):2713-2722..

(10) M Corrado and J-F Molinari. E_ects of residual stresses on the tensile fatigue behavior of concrete. Cement and Concrete Research 2016; 89:206-219.

(11) M Paggi and P Wriggers. A nonlocal cohesive zone model for_nite thickness interfaces Part I: mathematical formulation and validation with molecular dynamics. Computational Materials Science 2011; 50(5):1625-1633.

(12) M Paggi and P Wriggers. A nonlocal cohesive zone model for _nite thickness interfaces Part II: FE implementation and application to polycrystalline materials. Computational Materials Science 2011; 50(5):1634-1643.

(13) M Paggi, E Lehmann, C Weber, A Carpinteri, P Wriggers, and M Schaper. A numerical investigation of the interplay between cohesive cracking and plasticity in polycrystalline materials. Computational Materials Science 2013; 77:81-92.

(14) M Paggi and P Wriggers. Sti_ness and strength of hierarchical polycrystalline materials with imperfect interfaces. Journal of the Mechanics and Physics of Solids 2012; 60(4):557-572.

(15) HD Espinosa and PD Zavattieri. A grain level model for the study of failure initiation and evolution in polycrystalline brittle materials. Part II: Numerical examples. Mechanics of Materials 2003; 35(3-6):365-394. 
(16) M Ortiz and A Pandol_. Finite deformation irreversible cohesive elements for threedimensional crack-propagation analysis. International Journal for Numerical Methods in Engineering 1999; 44:1267-1282.

(17) T Luther and K_ Polycrystal models for the analysis of intergranular crack growth in metallic materials. Engineering Fracture Mechanics 2009; 76(15):2332-234.

(18) CV Verhoosel and MA Gutierrez. Modelling inter- and transgranular fracture in piezoelectric polycrystals. Engineering Fracture Mechanics 200976(6):742-760.

(19) M Paggi, M Corrado, and MA Rodriguez. A multi-physics and multi-scale numerical approach to microcracking and power-loss in photovoltaic modules. Composite Structures 2013 95:630-638.

(20) A Corigliano, A Ghisi, G Langfelder, A Longoni, F Zaraga, and A Merassi. A microsystem for the fracture characterization of polysilicon at the micro-scale. European Journal of Mechanics - A/Solids 2011; 30(2):127-136.

(21) R Vayrette, M Galceran, M Coulombier, S Godet, J-P Raskin, and T Pardoen. Size dependent fracture strength and cracking mechanisms in freestanding polycrystalline silicon_lms with nanoscale thickness. Engineering Fracture Mechanics, 168, Part A: 2016; 190203.

(22) N Moës, J Dolbow, and T Belytschko. A finite element method for crack growth without remeshing. International journal for numerical methods in engineering 1999; 46(1):131-150. 